\section{SYNTHESES AND PROPERTIES OF KANAMYCIN C DERIVATIVES ACTIVE AGAINST RESISTANT BACTERIA}

Sir:

The enzymatic mechanism of resistance to aminoglycoside antibiotics has been explored by Umezawa. ${ }^{1,2)}$ Many clinically isolated strains of aminoglycosides-resistant bacteria produce enzymes which phosphorylate, adenylylate or acetylate specific positions of aminoglycoside antibiotics. Kanamycin and kanamycin $\mathrm{B}$ are inactivated by aminoglycoside $3^{\prime}$-phosphotransferases, 2"-nucleotidyltransferase or 6'acetyltransferases. Furthermore, it has been shown that the 3'-phosphotransferase-producing bacteria are inhibited by $3^{\prime}$-deoxy derivatives of these antibiotics and $2^{\prime \prime}$-nucleotidyltransferaseproducing bacteria by the $1-\mathrm{N}-[(S)-4-a m i n o-2-$ hydroxybutyryl] derivatives. However, the 6'modification of these antibiotics has not yet produced derivatives which inhibit all 6'-acetyltransferase-producing strains. ${ }^{3)}$ Kanamycin C isolated from a culture filtrate of Streptomyces kanamyceticus as a minor component has the $6^{\prime}$ hydroxyl in place of the $6^{\prime}$-amino group in kanamycin $\mathrm{B}$, and is not inactivated by $6^{\prime}$-acetyltransferases. In this communication, we report chemical conversion of kanamycin B and its deoxy derivatives into kanamycin $\mathrm{C}$ and its deoxy derivatives, and synthesis of their 1-N-[(S)-4amino-2-hydroxybutyryl] derivatives.

Kanamycin B was converted into kanamycin $\mathrm{C}$ (I) in good yield by the following route. The free amino groups of $6^{\prime}-\mathrm{N}-\mathrm{BOC}^{*}$-kanamycin B monohydrate ${ }^{4)}$ were acetylated with acetic anhydride in methanol at room temperature for 5 hours and the BOC group was removed in $90 \%$ trifluoroacetic acid at room temperature for 45 minutes. The 1,3,2',3'"-tetra- $\mathrm{N}$-acetylkanamycin B trifluoroacetate thus obtained was treated with sodium nitrite in $33 \%$ aqueous acetic acid for 1 hour at ice temperature and then for 2 hours at room temperature. The reaction mixture was concentrated to dryness and the residue was dissolved in $2 \mathrm{~N} \mathrm{NaOH}$. After refluxing the alkaline solution for 12.5 hours, $\mathbf{I}$ in the solution was adsorbed on a column of Amberlite CG-50 $\left(70 \% \mathrm{NH}_{4}{ }^{+}\right)$resin and eluted with $0.5 \mathrm{~N}$ ammonia. Rechromatography on a Amber-

* tert-Butyloxycarbonyl lite CG-50 $\left(\mathrm{NH}_{4}{ }^{+}\right)$column eluted with $0.05 \mathrm{~N}$, $0.1 \mathrm{~N}$ and then $0.2 \mathrm{~N}$ ammonia gave pure I in $49 \%$ yield.

The deoxy derivatives, 3 -deoxykanamycin $\mathrm{C}$ (II) and 3',4'-dideoxykanamycin C (III) were synthesized from 6'-N-BOC-3'-deoxykanamycin $\mathrm{B}^{* *}$ and $6^{\prime}-\mathrm{N}-\mathrm{BOC}-3^{\prime}, 4^{\prime}$-dideoxykanamycin $\mathrm{B}^{6)}$ by the method described above in $40 \%$ and $24 \%$ yields, respectively.

The 1-N-acyl derivatives with (S)-4-amino-2hydroxybutyric acid were synthesized from partially protonated forms of I, II and III without any protection of amino groups. In an aqueous solution at $\mathrm{pH} 6.5 \pm 0.2$ adjusted with $1 \mathrm{~N} \mathrm{HCl}$, I, II or III was acylated with 1.5 equivalents of the N-hydroxysuccinimide ester of N-BOC-(S)-4-amino-2-hydroxybutyric acid ${ }^{6)}$ in dimethylformamide at room temperature for 6 hours and then the BOC group was removed in $90 \%$ trifluoroacetic acid at room temperature for 1 hour to afford 1-N-[(S)-4-amino-2-hydroxybutyryl]-kanamycin C (IV), -3'-deoxykanamycin $\mathrm{C}(\mathbf{V})$ or $-3^{\prime}, 4^{\prime}$-dideoxykanamycin C (VI) in $8.0 \%$, $8.5 \%$ or $8.3 \%$ yield, respectively. These derivatives were purified by column chromatography on Amberlite CG-50 $\left(\mathrm{NH}_{4}^{+}\right)$resin eluted with $0.2 \mathrm{~N}$ and $0.5 \mathrm{~N}$ ammonia, and on silica gel (Mallinckrodt, CC-7) developed with a mixture of chloroform, methanol and $17 \%$ ammonia (1: $4: 2$ in volume). With the resin chromatography, unreacted I, II or III was recovered in $40 \%, 34 \%$ or $45 \%$ yield, respectively. From the silica gel chromatography, a positional isomer having weak biological activity, 3-N-[(S)-4amino-2-hydroxybutyryl]-kanamycin C(VII), -3'deoxykanamycin C (VIII) or -3',4'-dideoxykanamycin C (IX) was separated in $11.0 \%, 6.1 \%$ or $7.4 \%$ yield, respectively.

The properties of all derivatives described above are summarized in Table 1. The structures of the acyl derivatives were confirmed by PMR and acid hydrolysis ${ }^{6)}$. On the carbon-13 FourIER-transform NMR spectra of I, IV, V, VI and VII, the chemical shifts were assigned as shown in Table 2. ${ }^{\text {) }}$

The minimum inhibitory concentration of $\mathbf{I}$ and its five derivatives are shown in Table 3.

** 6'-N-BOC-3'-deoxykanamycin B (mp 142 $154^{\circ} \mathrm{C}$ (dec.), $[\alpha]_{\mathrm{D}}^{22}+111^{\circ}$ (c 1, water), $\mathrm{C}_{23} \mathrm{H}_{45} \mathrm{~N}_{5} \mathrm{O}_{11}$. $\mathrm{H}_{2} \mathrm{O}$ ) was prepared from $3^{\prime}$-deoxykanamycin $\mathrm{B}^{5}$ ) by the method reported in a previous paper. ${ }^{4}$ ) 
Table 1. The properties of kanamycin $\mathrm{C}$ derivatives

\begin{tabular}{c|c|c|c|c|c}
\hline \multirow{2}{*}{ Derivative } & mp (dec.) & {$[\alpha]_{\mathrm{D}}$ in $\mathrm{H}_{2} \mathrm{O}$} & Molecular formula* & \multicolumn{2}{|c}{ Rf on TLC** } \\
& & & & A \\
\hline II & $180 \sim 220^{\circ} \mathrm{C}$ & $+110^{\circ}$ at $26^{\circ} \mathrm{C}$ & $\mathrm{C}_{18} \mathrm{H}_{36} \mathrm{~N}_{4} \mathrm{O}_{10} \cdot \frac{1}{2} \mathrm{H}_{2} \mathrm{O}$ & 0.38 & 0.59 \\
III & $200 \sim 220^{\circ} \mathrm{C}$ & $+118^{\circ}$ at $26^{\circ} \mathrm{C}$ & $\mathrm{C}_{18} \mathrm{H}_{36} \mathrm{~N}_{4} \mathrm{O}_{9} \cdot \frac{1}{2} \mathrm{H}_{2} \mathrm{O}$ & 0.49 & 0.66 \\
IV & $167 \sim 180^{\circ} \mathrm{C}$ & $+91^{\circ}$ at $26^{\circ} \mathrm{C}$ & $\mathrm{C}_{22} \mathrm{H}_{43} \mathrm{~N}_{5} \mathrm{O}_{13} \cdot \mathrm{H}_{2} \mathrm{O}$ & 0.18 & 0.19 \\
V & $151 \sim 160^{\circ} \mathrm{C}$ & $+83^{\circ}$ at $27^{\circ} \mathrm{C}$ & $\mathrm{C}_{22} \mathrm{H}_{43} \mathrm{~N}_{5} \mathrm{O}_{12} \cdot \mathrm{H}_{2} \mathrm{O}$ & 0.22 & 0.24 \\
VI & $142 \sim 158^{\circ} \mathrm{C}$ & $+76^{\circ}$ at $28^{\circ} \mathrm{C}$ & $\mathrm{C}_{22} \mathrm{H}_{43} \mathrm{~N}_{5} \mathrm{O}_{11} \cdot \mathrm{H}_{2} \mathrm{O}$ & 0.31 & 0.30 \\
VII & $148 \sim 153^{\circ} \mathrm{C}$ & $+87^{\circ}$ at $24^{\circ} \mathrm{C}$ & $\mathrm{C}_{22} \mathrm{H}_{43} \mathrm{~N}_{5} \mathrm{O}_{13} \cdot \mathrm{H}_{2} \mathrm{O}$ & 0.21 & 0.28 \\
VIII & $136 \sim 144^{\circ} \mathrm{C}$ & $+80^{\circ}$ at $24^{\circ} \mathrm{C}$ & $\mathrm{C}_{22} \mathrm{H}_{43} \mathrm{~N}_{5} \mathrm{O}_{12} \cdot \mathrm{H}_{2} \mathrm{O}$ & 0.27 & 0.36 \\
IX & $135 \sim 147^{\circ} \mathrm{C}$ & $+78^{\circ}$ at $24^{\circ} \mathrm{C}$ & $\mathrm{C}_{22} \mathrm{H}_{43} \mathrm{~N}_{5} \mathrm{O}_{11} \cdot \mathrm{H}_{2} \mathrm{O}$ & 0.34 & 0.44 \\
\hline
\end{tabular}

* Satisfactory results of elemental analyses were obtained for all compounds.

** Thin-layer chromatography on Silica gel G (Merck, Art 5721) using solvent A; butanol - ethanol chloroform - 28\% ammonia (4:5:2:8 in volume) (kanamycin $\mathrm{C}: \mathrm{Rf} 0.31$ ) and solvent B; chloroform - methanol - 28\% ammonia - water (1: 4:2:1 in volume) (kanamycin C: Rf 0.52).

Table 2. Carbon-13 chemical shifts

\begin{tabular}{|c|c|c|c|c|c|}
\hline \multirow{2}{*}{ Carbon } & \multicolumn{5}{|c|}{ Chemical shift $(\delta)$} \\
\hline & I & IV & $\mathbf{V}$ & VI & VII \\
\hline 1 & 51.3 & 49.9 & 50.0 & 50.0 & 51.1 \\
\hline 2 & 36.2 & 33.7 & 33.6 & 33.1 & 34.2 \\
\hline 3 & 50.3 & 50.5 & $50.4 *$ & $50.4^{*}$ & 49.0 \\
\hline 4 & 87.9 & 87.4 & 87.2 & 87.1 & 81.2 \\
\hline 5 & 75.2 & 75.7 & 75.8 & 75.8 & 76.0 \\
\hline 6 & 88.5 & 81.1 & 81.5 & 81.5 & 88.1 \\
\hline $1^{\prime}$ & 101.3 & 101.4 & 100.5 & 101.0 & $100.8^{*}$ \\
\hline $2^{\prime}$ & 56.2 & 56.2 & $50.0^{*}$ & $50.6^{*}$ & 55.9 \\
\hline $3^{\prime}$ & 74.5 & 74.5 & 35.7 & 26.0 & 74.4 \\
\hline $4^{\prime}$ & 70.7 & 70.5 & 65.5 & 26.5 & 70.5 \\
\hline $5^{\prime}$ & 73.8 & 73.7 & 74.3 & 70.9 & 73.3 \\
\hline $6^{\prime}$ & 61.6 & 61.6 & 61.7 & 64.9 & 61.6 \\
\hline $1^{\prime \prime}$ & 100.7 & 99.2 & 99.2 & 99.2 & $100.2 *$ \\
\hline $2^{\prime \prime}$ & 72.6 & 72.4 & 72.4 & 72.3 & 72.6 \\
\hline $3^{\prime \prime}$ & 55.1 & 55.0 & 55.0 & 54.9 & 55.1 \\
\hline $4^{\prime \prime}$ & 70.0 & 70.0 & 70.1 & 70.0 & 70.0 \\
\hline $5^{\prime \prime}$ & 72.9 & 73.0 & 72.9 & 72.9 & 72.9 \\
\hline $6^{\prime \prime}$ & 61.2 & 61.2 & 61.2 & 61.2 & 61.1 \\
\hline $1^{\prime \prime \prime}$ & & 176.4 & 176.4 & 176.2 & 176.4 \\
\hline $2^{\prime \prime \prime}$ & & 70.7 & 70.6 & 70.6 & 70.5 \\
\hline $3^{\prime \prime \prime}$ & & 35.2 & 35.3 & 35.2 & 35.0 \\
\hline $4^{\prime \prime \prime}$ & & 38.0 & 37.9 & 37.9 & 37.6 \\
\hline
\end{tabular}

$\delta:$ ppm from TMS in $\mathrm{D}_{2} \mathrm{O}$ using dioxane $(\delta=$ $67.4 \mathrm{ppm}$ ) as the internal reference.

* Assignments within any vertical column may be reversed.

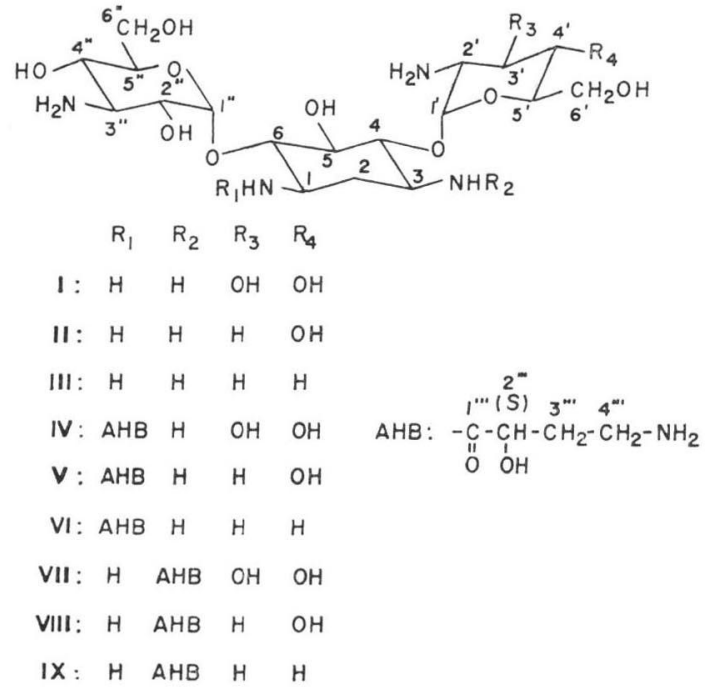

Among these derivatives, $\mathbf{V}$ is most active against all bacterial strains tested. This derivative showed $73 \%$ and $25 \%$ of the bacteriostatic activity of amikacin by the cylinder plate method using Bacillus subtilis PCI 219 and Escherichia coli JR66/W677, respectively, as the test organisms. These derivatives had low toxicity; when administered intravenously, mice tolerated a single dose of $400 \mathrm{mg} / \mathrm{kg}$ of II, IV or $\mathbf{V}$, but the same dose of III or VI caused death.

\section{SHINICHI Kondo \\ TSUYOSHI MIYASAKA \\ Kenzaburo Yoshida \\ KaTSUHARU InNUma \\ Hamao Umezawa}


Table 3. The antimicrobial spectra of kanamycin $\mathrm{C}$ derivatives

\begin{tabular}{|c|c|c|c|c|c|c|}
\hline \multirow{2}{*}{ Organisms } & \multicolumn{6}{|c|}{ Minimum inhibitory concentrations $(\mu \mathrm{g} / \mathrm{ml})$} \\
\hline & I & II & III & IV & $\mathbf{V}$ & VI \\
\hline Staphylococcus aureus FDA 209P & 6.25 & 3.13 & 12.5 & 6.25 & 6.25 & 12.5 \\
\hline Escherichia coli NIHJ & 6.25 & 12.5 & 25 & 6.25 & 6.25 & 12.5 \\
\hline Escherichia coli $\mathrm{K}-12$ & 12.5 & 12.5 & 50 & 12.5 & 6.25 & 12.5 \\
\hline Escherichia coli $\mathrm{K}-12 \mathrm{R} 5^{\mathrm{u}}$ & 6.25 & 12.5 & 25 & 6.25 & 6.25 & 12.5 \\
\hline Escherichia coli $\left.\mathrm{K}-12 \mathrm{ML} 1629^{\mathrm{b}}\right)$ & $>100$ & 12.5 & 25 & 6.25 & 6.25 & 6.25 \\
\hline Escherichia coli $\mathrm{K}-12 \mathrm{ML} 1630^{\mathrm{b}}$ ) & $>100$ & 12.5 & 25 & 12.5 & 12.5 & 12.5 \\
\hline Escherichia coli $\mathrm{K}-12$ ML1410 & 25 & 12.5 & 25 & 25 & 6.25 & 25 \\
\hline Escherichia coli $\mathrm{K}-12$ ML1410 R81 ${ }^{\mathrm{b}}$ ) & $>100$ & 25 & 100 & 12.5 & 12.5 & 25 \\
\hline Escherichia coli $\mathrm{LA} 290 \mathrm{R} 55^{\mathrm{d}}$ ) & $>100$ & $>100$ & $>100$ & 12.5 & 12.5 & 12.5 \\
\hline Escherichia coli LA290 R56 & 50 & 100 & $>100$ & 6.25 & 6.25 & 6.25 \\
\hline Escherichia coli LA290 R64 & 50 & 50 & $>100$ & 12.5 & 3.13 & 12.5 \\
\hline Escherichia coli $\mathrm{W} 677$ & 12.5 & 6.25 & 12.5 & 12.5 & 6.25 & 12.5 \\
\hline Escherichia coli JR66/W677 $7^{\mathrm{e}, \mathrm{d})}$ & $>100$ & $>100$ & $>100$ & 25 & 12.5 & 25 \\
\hline Klebsiella pneumoniae PCI 602 & 6.25 & 6.25 & 50 & 3.13 & 3.13 & 6.25 \\
\hline Klebsiella pneumoniae $22 \# 3038^{c, d)}$ & $>100$ & $>100$ & $>100$ & 12.5 & 6.25 & 25 \\
\hline Pseudomonas aeruginosa $\mathrm{A} 3$ & $>100$ & 12.5 & $>100$ & 12.5 & 6.25 & 12.5 \\
\hline Pseudomonas No. 12 & $>100$ & 100 & $>100$ & 50 & 50 & $>100$ \\
\hline Pseudomonas aeruginosa $\left.\mathrm{H}^{\circ}\right)$ & $>100$ & 100 & $>100$ & $>100$ & 100 & $>100$ \\
\hline Pseudomonas aeruginosa TI-13 $3^{\mathrm{b}}$ ) & $>100$ & 50 & $>100$ & 50 & 50 & $>100$ \\
\hline Pseudomonas aeruginosa $\mathrm{GN} 315^{\mathrm{a})}$ & $>100$ & 50 & $>100$ & 50 & 50 & $>100$ \\
\hline Pseudomonas aeruginosa 99 ) & $>100$ & 100 & $>100$ & 100 & 100 & $>100$ \\
\hline Pseudomonas aeruginosa $\mathrm{H} 11$ & $>100$ & 50 & $>100$ & 100 & 50 & $>100$ \\
\hline
\end{tabular}

Resistance mechanisms: a) 6'-acetyltransferase, b) 3'-phosphotransferase I, c) 3'-phosphotransferase II, d) 2"-nucleotidyltransferase, e) 3-acetyltransferase.

Institute of Microbial Chemistry Kamiosaki 3-Chome, Shinagawaku, Tokyo, Japan

(Received October 5, 1977)

\section{References}

1) Umezawa, H.: Biochemical mechanism of resistance to aminoglycosidic antibiotics. Advances in Carbohydrate Chemistry and Biochemistry. Vol. 30 (ed. by R. S. TIPSON \& D. Horton), pp. 183 225, Academic Press, New York, 1974

2) Umezawa, H.: Biochemical mechanism of resistance to aminoglycosidic antibiotics. Drug Action and Drug Resistance in Bacteria. 2. Aminoglycoside Antibiotics (ed. by S. MitsuHASHI), pp. $211 \sim 248$, University of Tokyo Press, Tokyo, 1975

3) Yagisawa, M.; S. Kondo, T. Takeuchi \& H. Umezawa: Aminoglycoside 6'-N-acetyltrans- ferase of Pseudomonas aeruginosa: Structural requirements of substrate. J. Antibiotics 28: $486 \sim 489,1975$

4) Kondo, S.; K. Iinuma, M. Hamada, K. Maeda \& H. Umezawa: Syntheses of isoseryl derivatives of kanamycins and their antibacterial activities. J. Antibiotics 27: 90 93, 1974

5) Takagi, Y.; T. Miyake, T. Tsuchiya, S. UmeZAWA: Synthesis of 3'-deoxykanamycin B. J. Antibiotics 26: 403 406, 1973

6) Kondo, S.; K. Iinuma, H. Yamamoto, K. Maeda \& H. Umezawa: Syntheses of 1-N$[(S)$-4-amino-2-hydroxybutyryl]-kanamycin B and $-3^{\prime}, 4^{\prime}$-dideoxykanamycin $\mathrm{B}$ active against kanamycin-resistant bacteria. J. Antibiotics 26: $412 \sim 415,1973$

7) See, for instance, Koch, K. F.; J. A. Rhoades, E. W. Hagaman \& E. Wenkert: Carbon-13 nuclear magnetic resonance spectral analysis of tobramycin and related antibiotics. J. Am. Chem. Soc. 96: 3300 3305, 1974 